\title{
Improvising Network life time of Wireless sensor networks using mobile data aggregator
}

\author{
K.Gangarajam \\ Vardhaman College of Engineering \\ Hyderabad, India
}

\author{
Eliyaz Mahammad \\ Vardhaman College of Engineering \\ Hyderabad, India
}

\begin{abstract}
Energy consumption becomes a primary concern in a Wireless Sensor Network. To pursue high energy saving at sensor nodes, a mobile collector should traverse the transmission range of each sensor in the field such that each data packet can be directly transmitted to the mobile collector without any relay.
\end{abstract}

Keywords: Wireless sensor networks (WSN); sensor; data sink; handover; polling point.

\section{INTRODUCTION}

In wireless sensor networks (WSNs) a large number of sensors scatter over a surveillance field and extract data of interests by reading real-world phenomena from the physical environment. Since sensors are typically battery-powered and left unattended after the initial deployment, it is generally infeasible to replenish the power supplies once they deplete the energy. Thus, energy consumption becomes a primary concern in a WSN, as it is crucial for the network to functionally operate for an expected period of time.

\section{RELATED WORK}

[1] In this paper, author proposed a new energy-efficient approach for clustering nodes in ad hoc sensor networks. Based on Hybrid Energy-Efficient Distributed clustering, which periodically selects cluster heads according to a hybrid of their residual energy and secondary parameter, such as nude proximity to its neighbors or node degree

Pros and Cons:

This method can be applied to the design of several types of sensor network protocols that require energy efficiency, scalability, prolonged network lifetime, and load balancing. Only provided a protocol for building a single cluster layer.

[2] In this paper, author first present how to place SNs by use of a minimal number to maximize the coverage area when the communication radius of the $\mathrm{SN}$ is not less than the sensing radius, which results in the application of regular topology to WSNs deployment.

Pros and cons:

WSN topology lifetime can extend by more than eight times on average by the mobile node rotation which is significantly better than existing alternatives.

It considers WSNs that are mostly static with a small number of mobile relays not practically declared for Dynamic WSNs.

[3] This paper deals with mobile data gathering in the sensor network which employs one or more mobile collectors that are robots or vehicles equipped with powerful transceivers and batteries.

Pros and cons:

The performance metrics observed are the data success rate (the fraction of generated data that matches the access points) and the required buffer capacities of the sensors and the MULEs.
An important issue that is not addressed in this paper i.e. latency.

[4] In this paper author presented the design and analysis of novel protocols that can dynamically configure a network to achieve guaranteed degrees of coverage and connectivity. Proposed work differs from existing connectivity or coverage maintenance protocols in several key ways.

Pros and cons:

Guaranteed connectivity and coverage configurations through both geometric analysis and extensive simulations can be provided which is the capability of our protocols.

It is not extending solution to handle more sophisticated coverage models and connectivity configuration and develop adaptive coverage reconfiguration for energy-efficient distributed detection and tracking techniques.

[5] In this paper author have developed an embedded networked sensor architecture that merges sensing and articulation with adaptive algorithms that are responsive to both variability in environmental phenomena discovered by the mobile sensors and to discrete events discovered by static sensors.

Pros and cons:

They also showed relationship among sampling methods, event arrival rate, and sampling performance are presented. Sensing diversity does not introduced which is used to enhance Fidelity Driven Sampling

\section{ALGORITHM}

Step 1: Initial setup is to design the network as less hop count transmission.

Step 2: Design a pp from the sensor devices (here we are setting PP which can receive the data from number of nodes).

Step3: if sensor having the data, then sensor finding the PP, which is near to that sensor.

Step 4: if sensor found any PP point node is available then transfers data to PP

Step 5: if PP has more data then it informs to control station.

Step 6: control station receives the number of control information from different PP's.

Step 7: after collecting the control message, CS makes the shortest route to collect the data from PP's. 
Step 8: MC moves towards each PP's and collects the info and returns back to $\mathrm{CS}$.

\section{MODULES}

There are five modules in this section. Those are

I. Analyzing the data sink details

II. Setting less hop count transmission

- Problem in static forward node

- Dynamic forward node

III. Select sensor as pp

- $\quad$ Static P

- Dynamic PP

IV. Find and collect data from pp's

V. Handover the data o BS.

\subsection{Analyzing the data sink details}

Handover the data to data sink when data sink within the transmission coverage area of sensors. The sensors which are located in the range of data sink it transforms all the information to the data sink with minimum hops.

\subsection{Setting Less Hop Count Transmission}

Multi-hop routing, packets have to experience multiple relays before reaching the data sink. Minimizing energy consumption on the forwarding path does not necessarily prolong network lifetime as some popular sensors on the path. So to avoid the problem in multi-hop routing we are setting the less hop count transmission.

\subsubsection{Static forward node:}

When the node forwarding the data continuously, then that node will loss more energy. It may causes node failure.

\subsubsection{Dynamic forward node:}

If the forward node is dynamically changed with less hop count node then energy loss of node should be very less.

So, In the first path the hop count is 3 where as the hop couny for the second path is 2 , Therefore for data transmission the preferable path is second path.

\subsection{Select Sensor as PP}

The selected polling points are the subset of sensors, each aggregating the local data from its affiliated sensors within a certain number of relay hops.

These PPs will temporarily store the data and upload them to the mobile collector when it arrives.

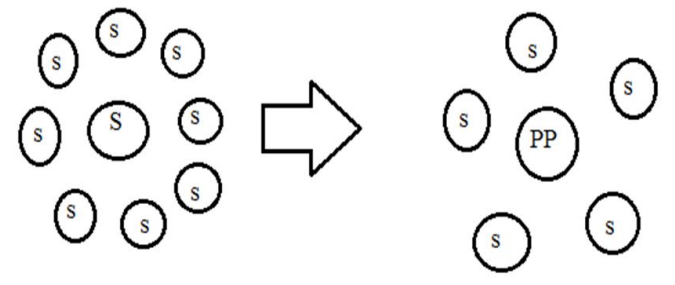

Figure 1: a) Group of sensors b) Group of sensors with polling point.
The PPs which are selected can simply be a subset of sensors in the network or some other special devices, such as storage nodes with larger memory and more battery power.

From a group of sensors one sensor will be elected as a polling point, which receives and send the information to the sensors.

\subsection{Find and Collect Data from Pp's}

Because of the freedom of mobile collector to move to any location in the sensing field, it provides an opportunity to plan an optimal tour for it.

Our main idea is first to find a set of special nodes referred to as PPs in the network and then determine the tour of the mobile collector by visiting each PP in a specific sequence.

When the mobile collector arrives it polls each PP to request data uploading and then it upload the data to MC.

The Polling points collect the information from all the sensors and that aggregated information is collected by the Mobile collector.

\subsection{Handover the Data to BS}

A Polling Point uploads the data packets to the mobile collector in a single hop.

Mobile collector begins its tour from the static data sink, which is located either inside or outside the sensing field, and collects data packets at the PPs and then returns the data to the data sink.

Finally MC Handover the data to data sink, such as BS.

The Mobile collectors move through all the polling points and collect the information and send it to Base Station

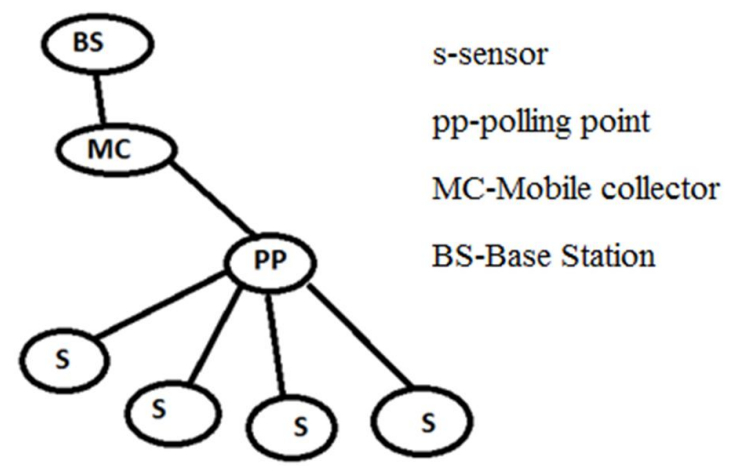

Figure 2: Mobile hierarchy

\section{RESULTS}

We did our research analysis in WSN by using NS2. In Ns2 we can show two type of output, one is Nam window and another one is $\mathrm{x}$ graph. 
In this paper, we showed our model testing output. From this model result, we can conclude our proposed method is better than previous one.

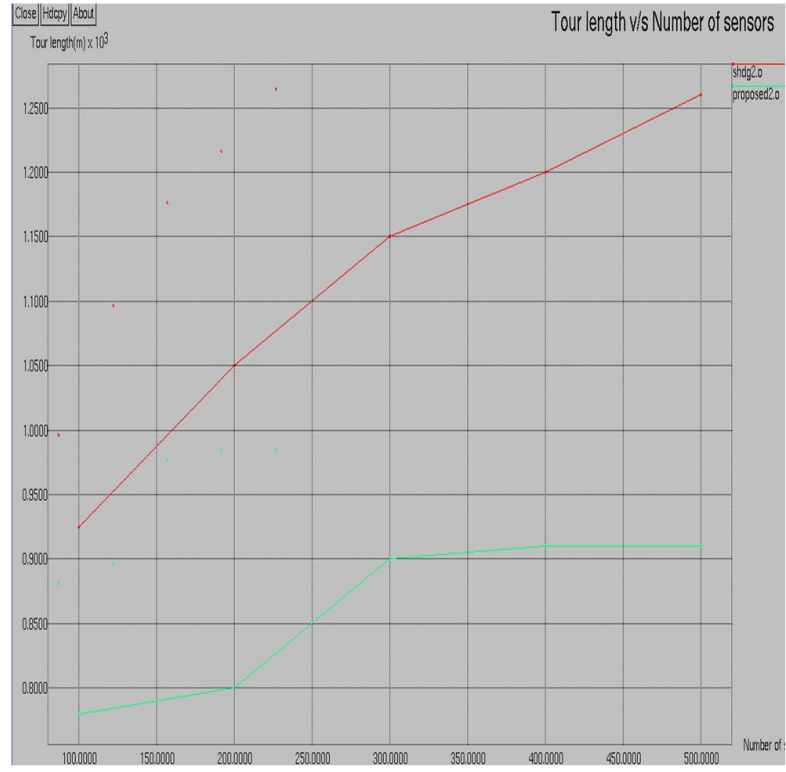

Figure 3: Tour length of proposed system and existing

From above graph, we got the result as we can avoid unnessary travalling time.

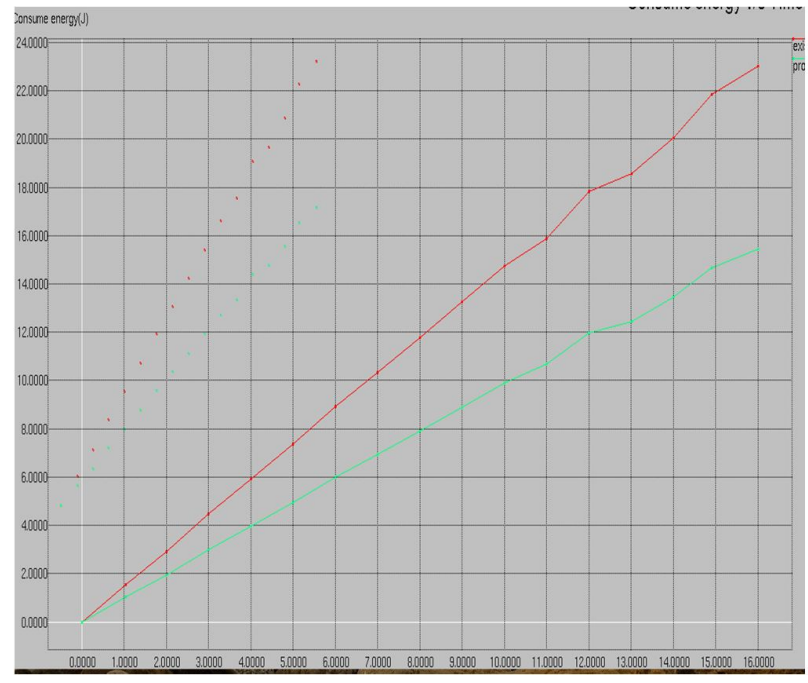

Figure 4: Energy comparison b/w proposed and existing

From this model result, we improved energy level and we reduced the energy consumption

From Nam window result we can see the process of our proposed model (data transmission, mobile collector movement)

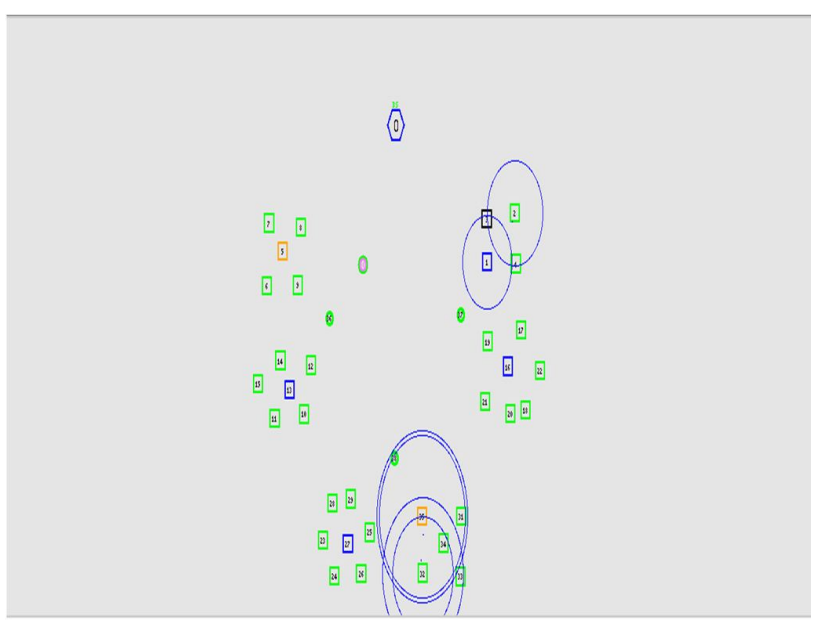

Figure 5: Nam window result mobile collector travelling

\section{CONCLUSION}

In this paper, we studied mobile data collection in wireless sensor networks by researching the tradeoff between the relay hop count of sensors for local data aggregation and the travel length of the mobile data aggregator. We proposed a pollingbased scheme and formulated it into the problem, then presented two efficient algorithms to give practically good results. Extensive simulations have been carried out to validate the efficiency of the system.

\section{REFERENCES}

1) Distributed Clustering in Ad-hoc Sensor networks: A Hybrid, Energy-Efficient Approach- Ossaia Younis and Sonia Fahmy - 2004.

2) Maximizing Networking Lifetime in Wireless Sensor Networks with Regular Topologies-HuiTian, Hong Shen and Matthew Roughan - 2008.

3) Modeling a Three-Tier Architecture for Sparse Sensor Networks- (R. Shah, S. Roy, S. Jain, and W. Brunette)-Sept. 2003

4) Integrated Coverage and Connectivity Configuration for Energy Conservation in Sensor Network-Guoliang xing, xiaorui wang, yuanfang zhang, chenyang lu, robert pless, and christopher gill -2005 .

5) Call and Response: Experiments in Sampling the Environment-Maxim A. Batalin, Mohammad Rahimi1, Yan $\mathrm{Yu}-2004$.

6) Data Storage Placement in Sensor Networks-Bo Sheng, Qun Li, Weizhen Mao - 2010. 
International Journal of Computer Applications Technology and Research

Volume 3- Issue 4, 273 - 276, 2014, ISSN: 2319-8656

7) Data Pre-Forwarding for Opportunistic Data Collection in Wireless Sensor Networks - Xiuchao Wu, Kenneth N. Brown 2012.

8) Mobile Relay Configuration in Data-intensive Wireless Sensor Networks-Fatme El-Moukaddem, Eric Torng, Guoliang Xing - 2013.
9) Novel Strategy for Data Dissemination to Mobile Sink Groups in Wireless Sensor Networks - Soochang Park, Euisin Lee, Min-Sook Jin, and Sang-Ha Kim - 2010.

10) Ubiquitous Data Collection for Mobile Users in Wireless Sensor Networks - Zhenjiang Li, Jiliang Wang, Zhichao Cao 2011. 